\title{
Generation of Functional Inhibitory Synapses Incorporating Defined Combinations of GABA(A) or Glycine Receptor Subunits
}

\author{
Christine L. Dixon ${ }^{1+}$, Yan Zhang' ${ }^{1}$ and Joseph W. Lynch ${ }^{1,2 *}$ \\ ${ }^{1}$ Queensland Brain Institute, University of Queensland, Brisbane, QLD, Australia, ${ }^{2}$ School of Biomedical Sciences, University \\ of Queensland, Brisbane, QLD, Australia
}

Fast inhibitory neurotransmission in the brain is mediated by wide range of $G_{A B A_{A}}$ receptor $\left(G A B A_{A} R\right)$ and glycine receptor $(G l y R)$ isoforms, each with different physiological and pharmacological properties. Because multiple isoforms are expressed simultaneously in most neurons, it is difficult to define the properties of individual isoforms under synaptic stimulation conditions in vivo. Although recombinant expression systems permit the expression of individual isoforms in isolation, they require exogenous agonist application which cannot mimic the dynamic neurotransmitter

OPEN ACCESS

Edited by: Kirsten Harvey, University College London, UK

Reviewed by:

Jochen C. Meier, Technical University Braunschweig, Germany

Piotr Bregestovski, Aix-Marseille University, France

*Correspondence: Joseph W. Lynch

j.lynch@uq.edu.au

${ }^{\dagger}$ Present address:

Christine L. Dixon, Institute of Neurology, University College London,

London, UK

Received: 12 November 2015 Accepted: 07 December 2015 Published: 23 December 2015

Citation:

Dixon CL, Zhang Y and Lynch JW (2015) Generation of Functional Inhibitory Synapses Incorporating Defined Combinations of $G A B A(A)$ or

Glycine Receptor Subunits.

Front. Mol. Neurosci. 8:80 doi: 10.3389/fnmol.2015.00080 profile characteristic of native synapses. We describe a neuron-HEK293 cell coculture technique for generating inhibitory synapses incorporating defined combinations of $\mathrm{GABA}_{A} R$ or GlyR subunits. Primary neuronal cultures, prepared from embryonic rat cerebral cortex or spinal cord, are used to provide presynaptic GABAergic and glycinergic terminals, respectively. When the cultures are mature, HEK293 cells expressing the subunits of interest plus neuroligin 2A are plated onto the neurons, which rapidly form synapses onto HEK293 cells. Patch clamp electrophysiology is then used to analyze the physiological and pharmacological properties of the inhibitory postsynaptic currents mediated by the recombinant receptors. The method is suitable for investigating the kinetic properties or the effects of drugs on inhibitory postsynaptic currents mediated by defined $\mathrm{GABA}_{A} \mathrm{R}$ or GlyR isoforms of interest, the effects of hereditary disease mutations on the formation and function of both types of synapses, and synaptogenesis and synaptic clustering mechanisms. The entire cell preparation procedure takes $2-5$ weeks.

Keywords: inhibitory postsynaptic current, IPSC, GABAergic, glycinergic, neuropharmacology, synaptogenesis, electrophysiology

\section{INTRODUCTION}

The central nervous system is comprised of circuits of interconnected neurons that serve to process specific types of information. These circuits regulate their own output by feedback and feedforward connections. Knowledge of the physiological properties of the inhibitory and excitatory synapses that mediate these connections is crucial for understanding the electrical behavior of circuits and ultimately of brain function. Fast inhibitory neurotransmission in these circuits is mediated by GABA type-A receptor $\left(\mathrm{GABA}_{\mathrm{A}} \mathrm{R}\right)$ and glycine receptor (GlyR) chloride channels. 
$\mathrm{GABA}_{\mathrm{A}}$ Rs exhibit a particularly broad range of heterogeneity. As members of the pentameric ligand-gated ion channel (pLGIC) family, five subunits are required to form a single functional oligomer. There are $19 \mathrm{GABA}_{\mathrm{A}} \mathrm{R}$ genes $(\alpha 1-6, \beta 1-3, \gamma 1-3, \delta$, $\varepsilon, \theta, \pi$, and $\rho 1-3)$ with the most common synaptic isoform comprising $\alpha 1, \beta 2$, and $\gamma 2$ subunits in a 2:2:1 stoichiometry. Although many hundreds of other subunit combinations are theoretically possible, it is thought that around one hundred exist naturally in the brain (Olsen and Sieghart, 2009). GlyRs exhibit far less diversity with only four genes $(\alpha 1-3, \beta)$ in humans (Lynch, 2009). They also belong to the pLGIC family and synaptic GlyR isoforms comprise heteromeric assemblies of $\alpha$ and $\beta$ subunits in a 2:3 or 3:2 stoichiometry (Durisic et al., 2012; Yang et al., 2012).

Each $\mathrm{GABA}_{\mathrm{A}} \mathrm{R}$ or GlyR isoform has a unique physiological and pharmacological profile and it is the unique properties of a particular isoform that are important for the appropriate functioning of a particular network. Disruptions to these properties can result in neurological disorders. For example, hereditary mutations that affect the function of $\mathrm{GABA}_{\mathrm{A}} \mathrm{Rs}$ or GlyRs can lead to epilepsy (Macdonald et al., 2010) or human hyperekplexia (Bode and Lynch, 2014), respectively. Other disruptive mechanisms are also possible. For example, a post-transcriptional RNA editing mechanism that is upregulated in temporal lobe epilepsy increases the prevalence of $\alpha 3$ GlyRs incorporating the P185L mutation (Meier et al., 2005; Eichler et al., 2008, 2009). Finally, a range of neurological disorders is known to result from aberrant changes to pLGIC phosphorylation status (Talwar and Lynch, 2014). For example, chronic pain sensitization is caused by prostaglandin-induced phosphorylation of $\alpha 3$ GlyRs (Harvey et al., 2004; Zeilhofer, 2005; Lynch and Callister, 2006) and ethanol-induced phosphorylation of the $\gamma 2 \mathrm{GABA}_{\mathrm{A}} \mathrm{R}$ subunit contributes to alcoholism (Qi et al., 2007).

Thus, characterizing the physiological and pharmacological properties of defined $\mathrm{GABA}_{\mathrm{A}} \mathrm{R}$ and GlyR isoforms under synaptic activation conditions is essential for understanding how neuronal circuits function in health and disease. However, it is difficult to study individual isoforms in their native neuronal environment due to the multitude of other isoforms present, and the difficulty in pharmacologically or genetically isolating the receptor isoform of interest. The neuron-HEK293 cell co-culture protocols we describe here solve this problem by providing a simple, efficient means of generating functional recombinant inhibitory synapses that selectively incorporate the recombinant $\mathrm{GABA}_{\mathrm{A}} \mathrm{R}$ or GlyR isoform of interest.

Co-culture approaches have previously been developed to understand the roles of synaptic adhesion molecules (including neurexin and neuroligin) in the formation of glutamatergic or GABAergic synapses (Scheiffele et al., 2000; Biederer et al., 2002; Dean et al., 2003; Graf et al., 2004; Sara et al., 2005; Kim et al., 2006; Dong et al., 2007; Fuchs et al., 2013) or to investigate the impact of disease-causing neuroligin mutations on GABAergic synaptogenesis (Chubykin et al., 2005; Sun et al., 2011). They have also been employed to characterize the functional properties of inhibitory post-synaptic currents (IPSCs), and have revealed kinetic differences among different $\mathrm{GABA}_{\mathrm{A}} \mathrm{R}$ isoforms (Wu et al., 2012; Dixon et al., 2014) and GlyR isoforms (Zhang et al., 2014).
The original co-culture protocol, involving postnatal hippocampal neurons and transfected HEK293 cells, was optimized for the immunohistochemical analysis of glutamatergic and GABAergic synapse development (Biederer and Scheiffele, 2007). A more recent protocol outlined an improved procedure for generating recombinant GABAergic synapses between striatal medium spiny GABAergic neurons and transfected HEK293 cells (Brown et al., 2014). However, this was also optimized for monitoring synapse development rather than for recording IPSCs in mature synapses. We have extended the co-culture approach in three ways. First, we describe the first spinal neuron-HEK293 cell co-culture preparation suitable for the efficient generation of recombinant glycinergic synapses. Second, we have simplified the technique for creating GABAergic synapses by using embryonic cortical neurons grown in serumfree media that does not promote the growth of glia (Brewer et al., 1993; Brewer, 1995). We have also have optimized the technique to facilitate the electrophysiological analysis of GABAergic and glycinergic IPSCs.

\section{MATERIALS AND METHODS}

\section{Overview}

Protocols for all procedures described in this study are detailed in the Supplementary Information. Lists of reagents and equipment are also provided. An overview of the coculture procedure is presented in Figure 1. The cerebral cortex contains large populations of GABAergic interneurons that were used to provide presynaptic GABAergic terminals onto HEK293 cells that recombinantly express the $\mathrm{GABA}_{\mathrm{A}} \mathrm{R}$ subunits of interest. Similarly, spinal neurons contain large populations of glycinergic interneurons that were used to provide glycinergic presynaptic terminals onto HEK293 cells expressing GlyR subunits of interest. The main steps in preparing the neuronal cultures are summarized in Figure 1 (blue box). The steps involved in HEK293 cell transfection are also described in Figure 1 (green box). An image of a GABAergic neuron forming synaptic contacts with HEK293 cell is shown in the inset.

\section{Preparation of Neuron Cultures}

Euthanasia of timed-pregnant rats was performed via $\mathrm{CO}_{2}$ inhalation, in accordance with procedures approved by the University of Queensland Animal Ethics Committee. To produce GABAergic interneuron cultures, E18 rat embryos were surgically removed from timed-pregnant rats and placed into chilled $\mathrm{Ca}$ Mg-free Hank's Balanced Salt Solution (CMF-HBSS) under sterile conditions. The cortical neuronal tissue was then pinched off using fine forceps, taking care to peel away the meninges to keep glial cell numbers down. The dissected neurons were then triturated, centrifuged and resuspended in Dulbecco's Modified Eagles Medium supplemented with 10\% fetal bovine serum (DMEM-FBS). To produce glycinergic interneuron cultures, E15 rat embryos were surgically removed and placed into ice cold CMF-HBSS under sterile conditions. The spinal cords were then removed and pinned at the wider proximal end while 


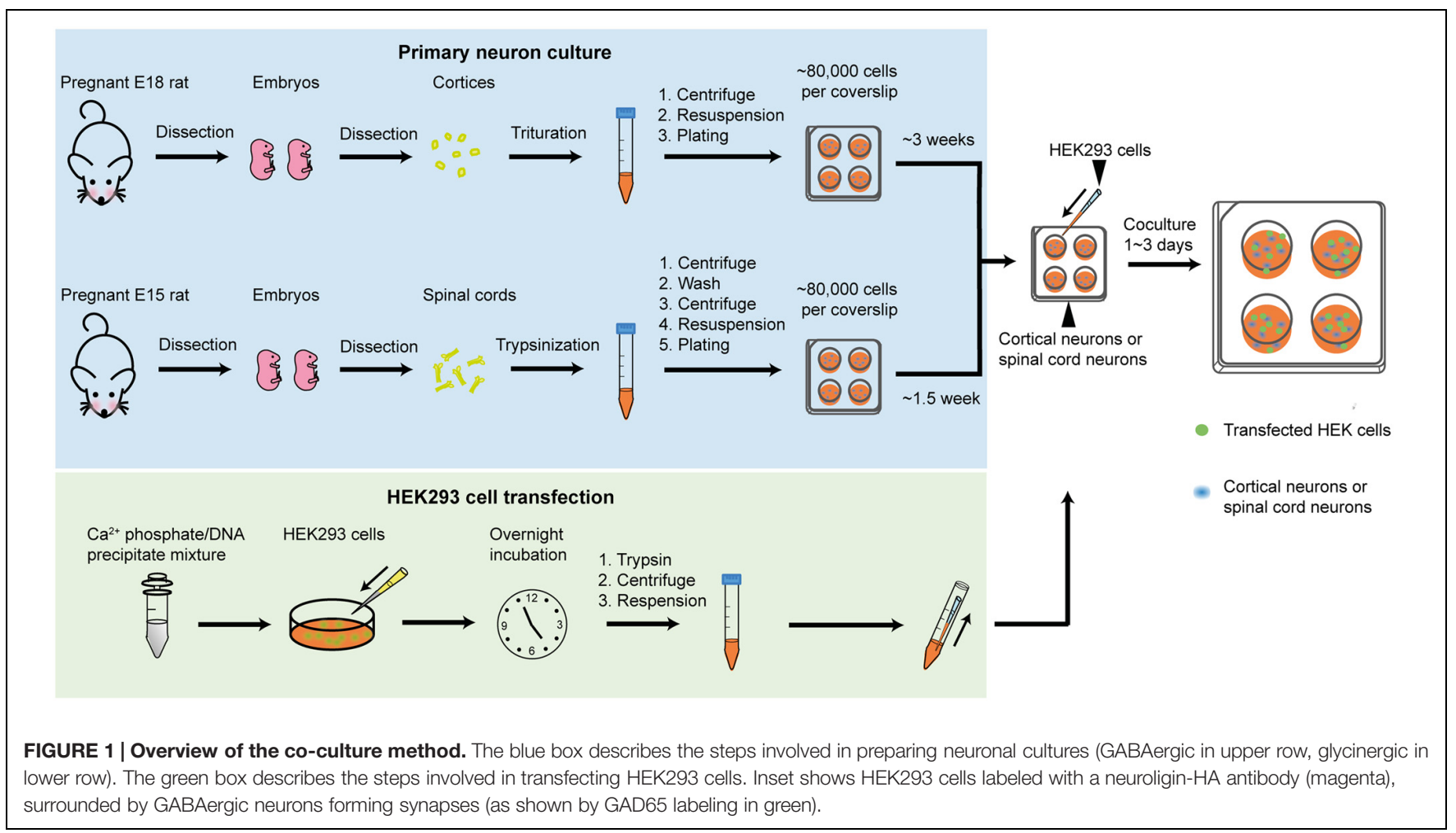

meninges were carefully detached. The dissected neurons were then triturated, centrifuged and resuspended in DMEM-FBS.

In both cases, the cells were then counted and between 40,000 and 80,000 neurons were plated onto each $12 \mathrm{~mm}$ poly-Dlysine-coated coverslip in four-well plates. As previously noted, neuronal density is a key consideration: if it is too low it impairs neuronal survival and if it is too high it encourages neuron clumping (Fuchs et al., 2013). Neuronal cultures were always maintained in a $5 \% \mathrm{CO}_{2}$ incubator at $37^{\circ} \mathrm{C}$. After $24 \mathrm{~h}$ the entire DMEM-FBS medium was replaced with Neurobasal medium including 2\% B27 and 1\% GlutaMAX supplements. A second (and final) feed 1 week later replaced half of this medium. In contrast to a previous protocol (Fuchs et al., 2013), we found that antibiotics were unnecessary. Neurons were used in coculture experiments between 3 and 6 weeks later (for GABAergic co-cultures) or 1-4 weeks later (for glycinergic co-cultures).

\section{Culture and Transfection of HEK293 Cells}

HEK293 cells were cultured in T75 flasks in DMEM-FBS and maintained in a $5 \% \mathrm{CO}_{2}$ incubator at $37^{\circ} \mathrm{C}$. The cells were passaged weekly. Prior to transfection, they were trypsinized and plated onto $35 \mathrm{~mm}$ culture dishes at a density of 5000 cells/dish. Following overnight incubation, the cells were transfected via a calcium phosphate co-precipitation protocol, using a total of $0.5-$ $2.5 \mu \mathrm{g}$ DNA per dish. Then, following incubation for $5-20 \mathrm{~h}$ in a $3 \% \mathrm{CO}_{2}$ incubator, the transfection was terminated by washing twice with divalent cation-free phosphate buffered saline. The cells were then trypsinized, centrifuged, and resuspended in Neurobasal medium (including 2\% B27 and 1\% GlutaMAX supplements) then seeded onto the neurons. One $35 \mathrm{~mm}$ dish of
HEK293 cells was sufficient to seed four coverslips of neurons. Once seeded with HEK293 cells, the co-cultures were returned to the incubator overnight to allow synapses to form. Cultures were used for patch clamp recording over the following 2-3 days.

\section{Plasmid DNA}

A total of $0.5-2.5 \mu \mathrm{g}$ plasmid DNA should be added to each $35 \mathrm{~mm}$ dish of HEK293 cells. This amount may vary according to the individual plasmid expression efficiency, the number and ratios of plasmids to be transfected and the transfection method. When using a calcium phosphate co-precipitation protocol, our recommendations are as follows. When expressing synaptic GlyRs, the total plasmid DNA should comprise: $0.2 \mu \mathrm{g}$ neuroligin $2 \mathrm{~A}, 0.2 \mu \mathrm{g}$ gephyrin, $0.1 \mu \mathrm{g}$ EGFP, with the remainder comprising GlyR subunit DNA that varies according to the number of subunits and the ratio of subunit DNA required. For example, when transfecting GlyR $\alpha$ subunits as homomers, add $0.5 \mu \mathrm{g}$ DNA. When transfecting $\alpha$ and $\beta$ GlyR subunits in $1: 10$ or $1: 50$ ratios add a total of $2 \mu \mathrm{g}$ DNA. We recommend transfecting $\alpha 1: \beta$, $\alpha 2: \beta$, and $\alpha 3: \beta$ subunits in $1: 50,1: 50$, and $1: 10$ ratios, respectively (Zhang et al., 2014). When expressing $\alpha 1 \beta 2 \gamma 2 \mathrm{GABA}_{\mathrm{A}} \mathrm{Rs}$, the $\alpha 1, \beta 2, \gamma 2$, EGFP and neuroligin 2A plasmid DNAs should be transfected in a 1:1:4:1:1 ratio with a combined total of $0.5 \mu \mathrm{g}$ DNA.

In experiments described in Figures 2 and 3 we employed plasmid DNAs encoding the human $\alpha 1$ (pCIS), rat $\alpha 3 \mathrm{~L}$ (pcDNA3.1), and human $\beta$ (pcDNA3.1) GlyR subunits, plus mouse neuroligin $2 \mathrm{~A}$ (pNice) and rat gephyrin (pCIS). In experiments described in Figure 4, we employed human $\alpha 2$ (pcDNA3.1), human $\alpha 4$ (pCIS), $\beta 2$ (pcDNA3.1), and $\gamma 2 \mathrm{~L}$ 


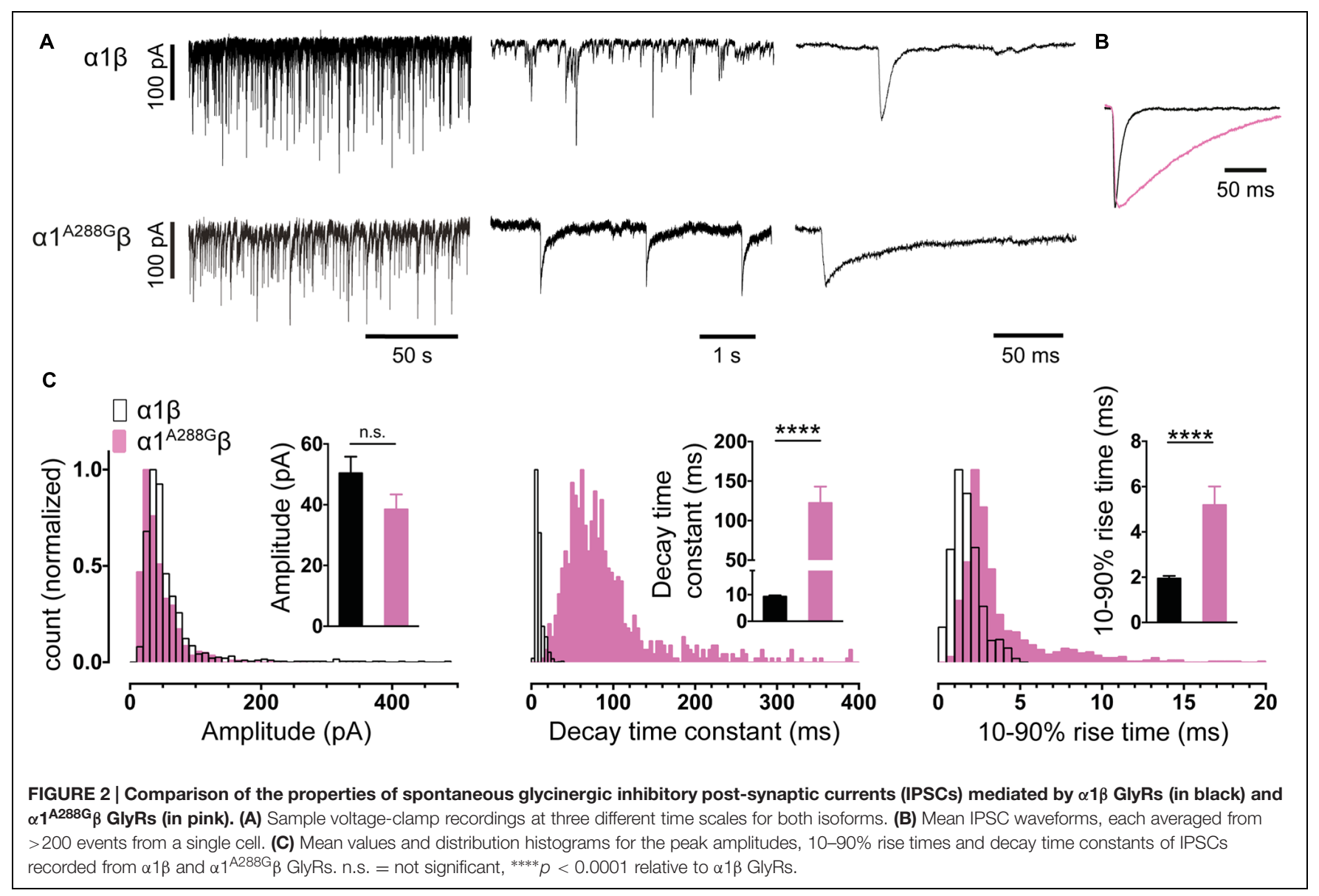

(pcDNA3.1) GABA $_{A} \mathrm{R}$ subunits. Site-directed mutagenesis was performed using the QuikChange mutagenesis kit (Agilent Technologies) according to manufacturers' instructions and the successful incorporation of mutations was confirmed by DNA sequencing.

\section{Patch Clamp Electrophysiology and Data Analysis}

Standard patch-clamp electrophysiology equipment can be used, with the only specific requirement being a fluorescence microscope for identifying GFP fluorescent cells. Coverslips containing the co-cultured cells were placed gently into the recording chamber on the microscope stage and perfused continuously with an extracellular solution comprising (in $\mathrm{mM}$ ): $140 \mathrm{NaCl}, 5 \mathrm{KCl}, 2 \mathrm{CaCl}_{2}, 1 \mathrm{MgCl}_{2}, 10 \mathrm{HEPES}$, and $10 \mathrm{D}$-glucose, adjusted to $\mathrm{pH} 7.4$ with $\mathrm{NaOH}$. Patch pipettes were filled with an intracellular solution containing (in $\mathrm{mM}$ ): $145 \mathrm{CsCl}, 2 \mathrm{CaCl}_{2}$, $2 \mathrm{MgCl}_{2}, 10 \mathrm{HEPES}, 10 \mathrm{EGTA}$, and $2 \mathrm{MgATP}$, adjusted to $\mathrm{pH}$ 7.4 with $\mathrm{NaOH}$. HEK293 cell selection is largely a matter of trial and error. A good starting point is to select large, strongly fluorescent green cells that are closely surrounded by many neurons, especially small clumps of neurons. Cells with a textured (rather than smooth) appearance often yield abundant IPSCs.

The electrophysiological techniques may vary according to the experimental requirements. For example, if precise quantitation of rise times is required, it is extremely important that the filtering and digitisation rates are high and that pipette series resistance is low to avoid artefactually slowing down the event. In contrast, testing the effect of a drug on IPSC decay rate is less sensitive to filtering, and it may be necessary to use higher resistance pipettes to obtain a membrane seal that is stable enough to permit recordings that are long enough to apply and wash out the drug.

In all experiments described below, series resistance was compensated to $60 \%$ of maximum and was monitored throughout the recording. Spontaneous and action potentialevoked IPSCs in HEK293 cells were recorded at a holding potential $-60 \mathrm{mV}$ and currents were filtered at $4 \mathrm{kHz}$ and sampled at $10 \mathrm{kHz}$. Only cells with a stable series resistance of $<25 \mathrm{M} \Omega$ throughout the recording period were included in the analysis. Patch pipettes (4-8 M $\Omega$ resistance) were made from borosilicate glass (GC150F-7.5, Harvard Apparatus). Analyses of IPSC amplitude, $10-90 \%$ rise time, and decay time constant (single-exponential) were performed using Axograph $\times$ (Axograph Scientific). Single peak IPSCs with amplitudes of at least three times above the background noise were detected using a semi-automated sliding template. Each detected event was visually inspected and only wellseparated IPSCs with no inflections in the rising or decay phases (suggestive of superimposed events) were included. The respective parameters from all selected events from a single 


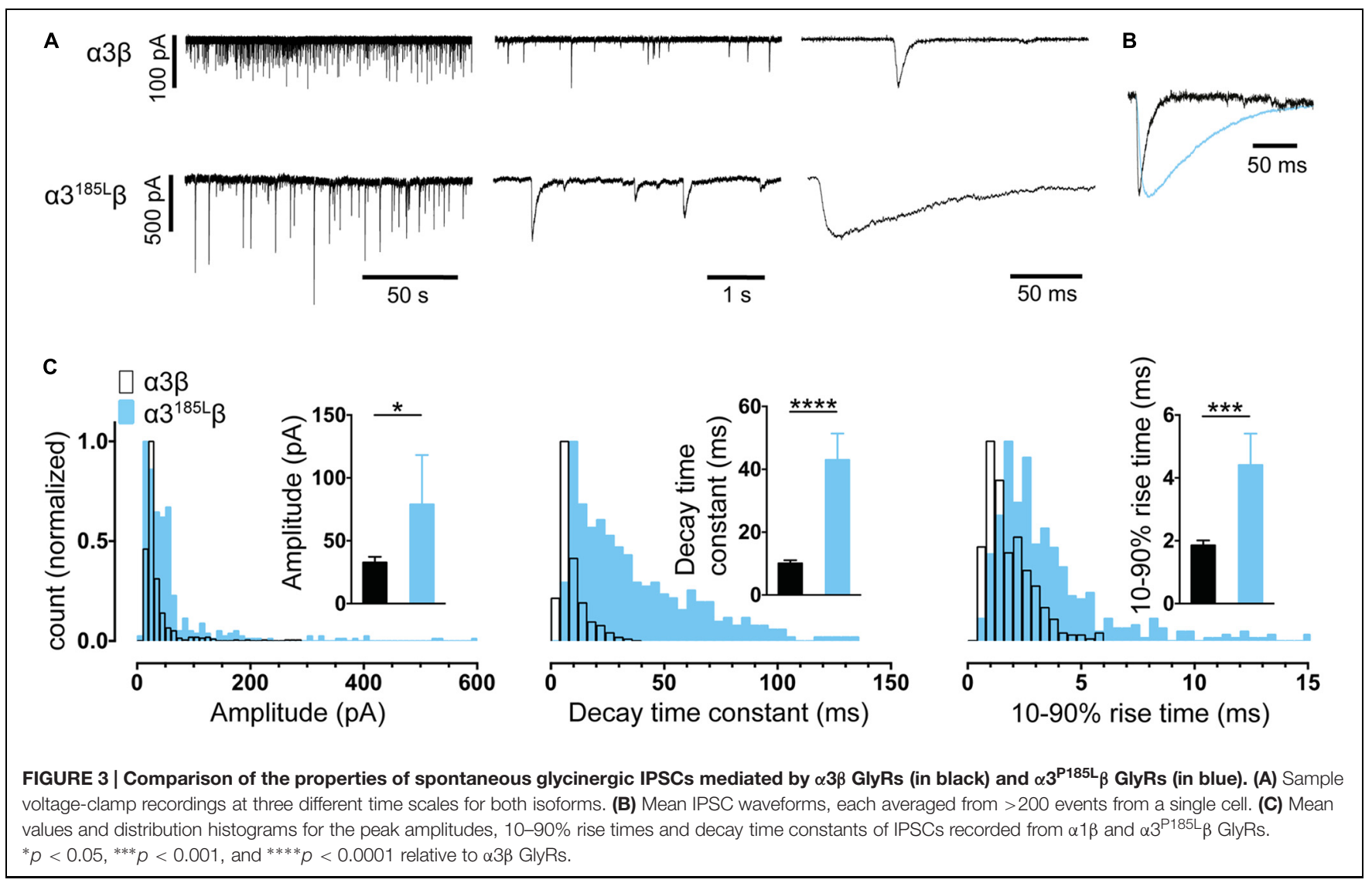

cell were averaged and are presented as a single data point in Figures 2-4. The averages from multiple cells were then pooled to obtain group data. Statistical analysis and plotting were performed on group data with Prism 5 (GraphPad Software). All data are presented as mean \pm SEM. One-way and two-way ANOVA were employed for multiple comparisons. For all tests, the number of asterisks corresponds to level of significance: ${ }^{*} p<0.05,{ }^{* *} p<0.01,{ }^{* * *} p<0.001$ and ${ }^{* * *} p<0.0001$.

\section{RESULTS}

\section{Glycinergic IPSCs}

While we found that some co-cultures exhibited spontaneous activity in almost all green fluorescent HEK293 cells, it was more typical to observe spontaneous glycinergic IPSCs in around 20\% of cells. This success rate is adequate for most experiments.

It is important to establish that the spontaneous IPSCs produced by the co-culture synapses exhibit similar characteristics to those mediated by native synapses incorporating the same subunits. Figure $\mathbf{2 A}$ shows sample IPSC recordings from HEK293 cells expressing $\alpha 1 \beta$ GlyRs. An IPSC averaged from $>200$ events recorded from multiple cells is shown in Figure 2B. Mean amplitudes, $10-90 \%$ rise times and decay time constants are presented in Figure 2C. Frequency distributions of IPSC amplitudes, $10-90 \%$ rise times and decay time constants all exhibit monotonic distributions suggesting a single functional population of synapses (Figure 2C). In adult hypoglossal motor neurons, where the $\alpha 1 \beta$ GlyR isoform predominates (Lynch, 2009), the 10-90\% rise times and decay time constants range between $0.6-1.8$ and $4.9-7.7 \mathrm{~ms}$, respectively (Singer et al., 1998; Graham et al., 2006; Hirzel et al., 2006; Muller et al., 2006). The mean decay time constant (9.3 ms) and the $10-90 \%$ rise time $(1.9 \mathrm{~ms})$ recorded in our co-culture synapses correspond well with these results.

We performed a similar analysis on $\alpha 3 \beta$ co-culture synapses and found the mean IPSC rise and decay times to be remarkably similar to those mediated by $\alpha 1 \beta$ GlyRs (Figures $\mathbf{3 A}-\mathbf{C}$ ). These parameters were also distributed monotonically, again suggesting a single population of synapses (Figure 3C). Although $\alpha 3 \beta$ mediated IPSCs have yet to be recorded in isolation in native neurons, evidence to date suggests their rise and decay times are indistinguishable from those mediated by $\alpha 1 \beta$ GlyRs (Harvey et al., 2004). This fits well with the results from our engineered synapses.

The $\alpha 1$ GlyR subunit D80A and A52S mutations result in startle disease phenotypes in mice (Graham et al., 2006; Hirzel et al., 2006). We previously demonstrated that engineered synapses incorporating $\alpha 1^{\mathrm{D} 80 \mathrm{~A}} \beta$ and $\alpha 1^{\mathrm{A} 52 \mathrm{~S}} \beta$ GlyRs exhibited accelerated IPSC decay rates closely resembling those recorded in native synapses from mutant mice homozygous for these mutations (Zhang et al., 2014). This provides an important validation of our technique. In this study we sought to determine whether GlyRs may be located both synaptically 

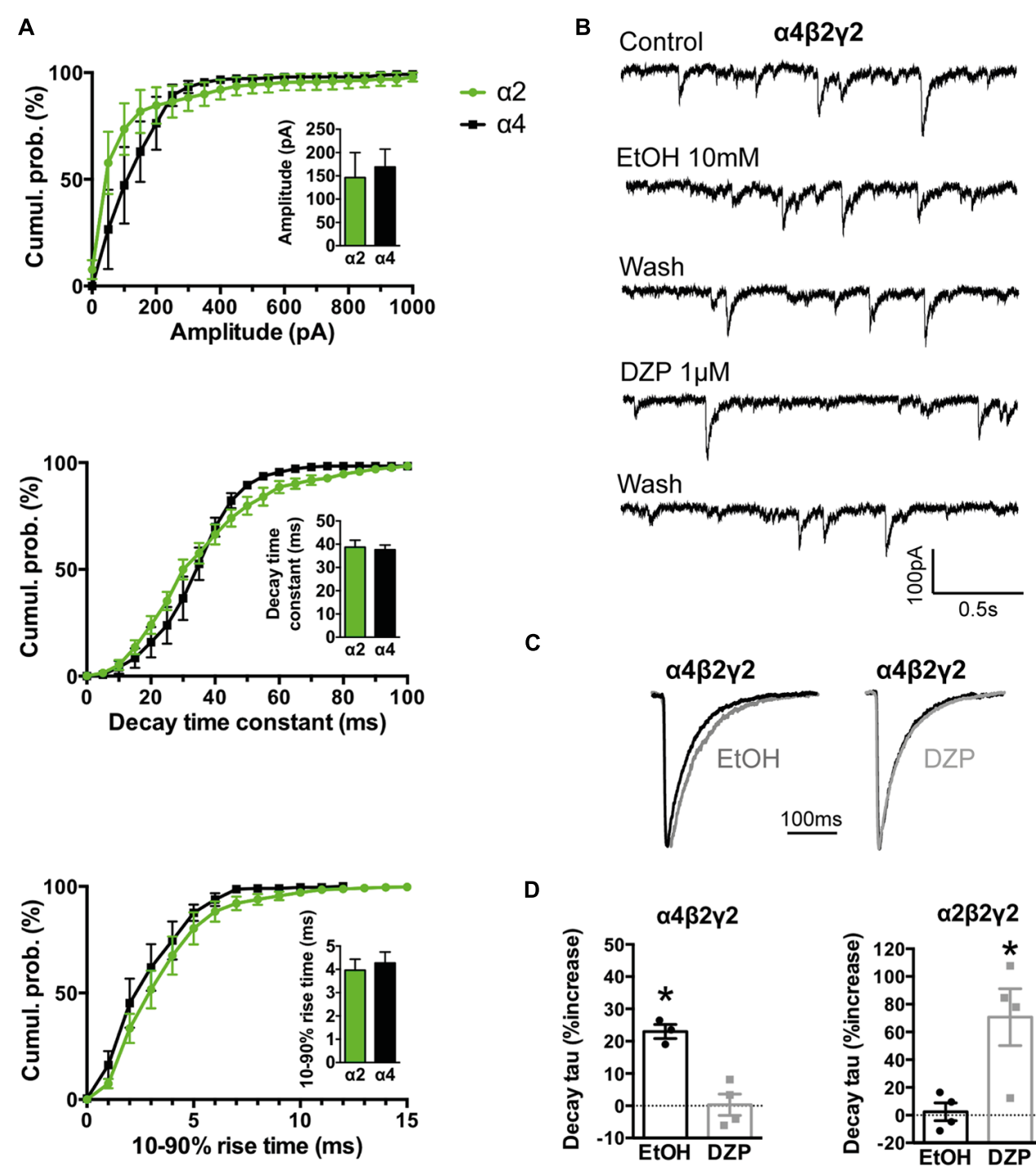

D

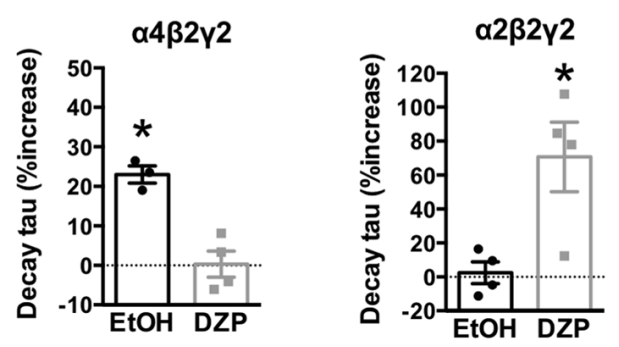

FIGURE 4 | Comparison of kinetics and pharmacological properties of IPSCs mediated by $\alpha \mathbf{2} \beta 2 \gamma 2$ and $\alpha \mathbf{4} \beta 2 \gamma 2$ GABA $A_{A}$ Rs. (A) Cumulative probability data averaged from four cells expressing $\alpha 4 \beta 2 \gamma 2 \mathrm{GABA}_{A}$ Rs were compared to previously published data for $\alpha 2 \beta 2 \gamma 2 \mathrm{GABA}_{A} \mathrm{Rs}(n=7$; Dixon et al., 2014). We found no significant differences in IPSC amplitudes, 10-90\% rise times or decay time constants. (B) Sample recordings of spontaneous IPSCs mediated by a $4 \beta 2 \gamma 2$ $\mathrm{GABA}_{A}$ Rs before and after the application of $10 \mathrm{mM}$ ethanol and $1 \mu \mathrm{M}$ diazepam. (C) Examples of mean IPSC waveforms mediated by $\alpha 4 \beta 2 \gamma 2 \mathrm{GABA} \mathrm{A}_{\mathrm{R}}$, each averaged from $>100$ events from a single cell, before and after the application of $10 \mathrm{mM}$ ethanol or $1 \mu \mathrm{M}$ diazepam. (D) The decay time constants of IPSCs mediated by $\alpha 4 \beta 2 \gamma 2 \mathrm{GABA}_{A} R s$ were significantly prolonged by $10 \mathrm{mM}$ ethanol but not by $1 \mu \mathrm{M}$ diazepam (left). In contrast, IPSCs mediated by $\alpha 2 \beta 2 \gamma 2 \mathrm{GABA} A \mathrm{Rs}$ were significantly prolonged by $1 \mu \mathrm{M}$ diazepam but not by $30 \mathrm{mM}$ ethanol (right). Diazepam data for $\alpha 2 \beta 2 \gamma 2 \mathrm{GABA}_{\mathrm{A}} \mathrm{Rs}$ were reproduced from (Dixon et al., 2014). ${ }^{*} p<0.05$ relative to drug-free control in same cell.

and peri-synaptically in HEK293 cells by introducing mutations that dramatically enhanced the glycine sensitivity. We reasoned that if GlyRs are located peri-synaptically then enhancing their glycine sensitivity may render them susceptible to activation by synaptically released glycine, and if so this should be detectable as an additional slow rise time component (Wu et al., 2012). As noted above, $\alpha 3^{\mathrm{P} 185 \mathrm{~L}}$ results from a post-transcriptional RNA editing mechanism that is upregulated in (and is causative of) human temporal lobe epilepsy (Eichler et al., 2008, 2009). This mutation reduces the glycine $\mathrm{EC}_{50}$ from 70.9 to $7.4 \mu \mathrm{M}$ (Legendre et al., 2009). We also investigated the $\alpha 1^{\mathrm{A} 288 \mathrm{G}}$ mutation (which is not associated with a disease) because it reduces the glycine $\mathrm{EC}_{50}$ from 30.9 to $6.0 \mu \mathrm{M}$ (Lynagh and Lynch, 2010).

As shown in Figures 2A-C, IPSCs mediated by $\alpha 1^{\mathrm{A} 288 \mathrm{G}} \beta$ GlyRs exhibited significantly slower rise times and decay time constants, although additional slow components were never observed on the rising phase of IPSCs. As with unmutated $\alpha 1 \beta$ GlyRs, these properties were monotonically distributed (Figure 2C) suggesting a single postsynaptic receptor population. Similarly, Figures 3A-C shows that IPSCs mediated by $\alpha 3^{\mathrm{P} 185 \mathrm{~L}} \beta$ GlyRs also exhibited significantly slower rise times and decay time constants that were distributed monotonically. Thus, we 
were not able to unequivocally distinguish a putative perisynaptic GlyR population in either case.

\section{GABAergic IPSCs}

As with glycinergic IPSCs, we typically observed GABAergic IPSCs in around 20\% of HEK293 cells. The rise times and decay time constants of IPSCs recorded from the dominant $(\alpha 1 \beta 2 \gamma 2)$ synaptic subtype (1.2 and $4.0 \mathrm{~ms}$, respectively) are in close accordance with those recorded from neurons known to predominantly express this subtype (Dixon et al., 2014). The co-culture system has revealed that other $\mathrm{GABA}_{\mathrm{A}} \mathrm{R}$ subunit combinations can yield IPSCs with dramatically different rise and decay times (Wu et al., 2012; Dixon et al., 2014), although it is as yet unclear how these properties relate to those of the same isoforms when expressed in native neuronal synapses.

We have previously demonstrated that the effects of some clinically important drugs on co-culture GABAergic synaptic IPSCs are similar to those recorded at corresponding neuronal synapses. For example, $1 \mu \mathrm{M}$ diazepam or $0.1 \mu \mathrm{M}$ flunitrazepam significantly increased the decay time constants of IPSCs mediated by $\alpha 2$-containing $\mathrm{GABA}_{\mathrm{A}} \mathrm{Rs}$ (Dixon et al., 2014) and $1 \mu \mathrm{M}$ zolpidem or $1 \mu \mathrm{M}$ eszopiclone increased IPSC magnitudes and decay time constants of IPSCs mediated by $\alpha 1$-containing $\mathrm{GABA}_{\mathrm{A}} \mathrm{Rs}$ (Dixon et al., 2015). Here we extended this characterisation by performing a 'reciprocal' pharmacological comparison of $\alpha 2 \beta 2 \gamma 2$ and $\alpha 4 \beta 2 \gamma 2 \mathrm{GABA}_{\mathrm{A}} \mathrm{Rs}$, based the knowledge that $\alpha 4$-containing $\mathrm{GABA}_{\mathrm{A}}$ Rs are highly sensitive to ethanol and insensitive to benzodiazepines, whereas $\alpha 2$-containing $\mathrm{GABA}_{\mathrm{A}} \mathrm{Rs}$ have the opposite profile (Knoflach et al., 1996; Wallner et al., 2006). As shown in Figures 4A,B, IPSCs mediated by recombinant $\alpha 2 \beta 2 \gamma 2$ and $\alpha 4 \beta 2 \gamma 2 \mathrm{GABA}_{\mathrm{A}} \mathrm{Rs}$ exhibit identical amplitudes, $10-90 \%$ rise times and decay time constants. A physiologically relevant $(10 \mathrm{mM})$ ethanol concentration significantly increased the IPSC decay time constant in $\alpha 4 \beta 2 \gamma 2 \mathrm{GABA}_{\mathrm{A}} \mathrm{Rs}$ whereas $1 \mu \mathrm{M}$ diazepam had no effect (Figures 4C,D). On the other hand, $1 \mu \mathrm{M}$ diazepam significantly prolonged the IPSC decay time constant in $\alpha 2 \beta 2 \gamma 2 \mathrm{GABA}_{\mathrm{A}} \mathrm{Rs}$ (Dixon et al., 2014), whereas even a very high $(30 \mathrm{mM})$ ethanol concentration had no effect (Figure 4D).

\section{DISCUSSION}

\section{Applications of the Protocol}

We have described protocols for reliably generating recombinant inhibitory synapses that incorporate defined GlyR or $\mathrm{GABA}_{\mathrm{A}} \mathrm{R}$ isoforms of interest. These are suitable for investigating (1) the kinetics of IPSCs mediated by defined $\mathrm{GABA}_{\mathrm{A}} \mathrm{R}$ or GlyR isoforms, (2) the effects of drugs on IPSCs mediated by defined $\mathrm{GABA}_{\mathrm{A}} \mathrm{R}$ or GlyR isoforms, (3) the effect of posttranslational modifications (e.g., phosphorylation) and hereditary disease mutations on the formation and function of both types of synapses, and (4) synaptogenesis and synaptic clustering mechanisms in both types of synapses. We now expand on each of these points.

\section{IPSC Kinetics}

Inhibitory post-synaptic currents mediated by different synaptic $\mathrm{GABA}_{\mathrm{A}} \mathrm{R}$ or GlyR isoforms exhibit unique physiological and pharmacological profiles. It is useful to quantitate these properties because they may help in identifying the presence, or even the role, of a particular isoform in a particular neuron and also because accurate parameters provide key inputs into computational models of neuron or network function. Although studying recombinant receptors in standard heterologous expression systems such as HEK293 cells or Xenopus oocytes allows the electrophysiological properties of a single isoform to be studied in isolation, this approach is limited because the neurotransmitter must be applied artificially and it cannot mimic the fast ( $\mu s)$ dynamic neurotransmitter concentration profile that exists in the synaptic cleft.

\section{Investigating Drug Efficacy and Selectivity}

The $\mathrm{GABA}_{\mathrm{A}} \mathrm{R}$ is an established therapeutic target for clinical indications including epilepsy, anxiolysis, muscle spasms, sedation and anesthesia. GABA $\mathrm{A}_{\mathrm{A}}$-targeted drugs currently in clinical use are not strongly subtype-selective and this can lead to dose-limiting side effects. For example, diazepam produces effective anxiolysis by positively modulating $\alpha 2$-containing $\mathrm{GABA}_{\mathrm{A}} \mathrm{Rs}$, although it also elicits the side effect of sedation by modulating $\alpha 5$-containing $\mathrm{GABA}_{\mathrm{A}} \mathrm{Rs}$ (Trincavelli et al., 2012). Drugs specific for other isoforms are also being sought. For example, selective modulators of $\alpha 5$-containing $\mathrm{GABA}_{\mathrm{A}} \mathrm{Rs}$ are being developed for a range of indications including stroke, cognitive impairment, and schizophrenia (Soh and Lynch, 2015). Although GlyRs are not currently targeted by clinically useful drugs, molecules that selectively enhance $\alpha 3$-containing GlyRs are considered promising as new generation treatments for chronic pain (Zeilhofer, 2005; Lynch and Callister, 2006). When evaluating new molecules as potential therapeutic lead compounds for synaptically localized receptors, it is important to test their potency, efficacy and subtype-selectivity under realistic synaptic activation conditions. The system we describe provides the most definitive means available of evaluating drug efficacy and selectivity at IPSCs mediated by defined receptor isoforms.

\section{Investigating Disease Mutations}

Engineered synapses have yet to be used to study diseasecausing $\mathrm{GABA}_{\mathrm{A}} \mathrm{R}$ mutations or modifications, and hence, the method has not realized its full potential as a model system for understanding the molecular pathology of neurological disorders. Mutations in $\mathrm{GABA}_{\mathrm{A}} \mathrm{R} \alpha 1$ and $\gamma 2$ subunits have long been associated with genetic epilepsy syndromes (Macdonald et al., 2010). Thus far, the function and pharmacology of epilepsycausing mutant $\mathrm{GABA}_{\mathrm{A}}$ Rs have only been investigated using whole-cell recordings of steady-state GABA-activated currents in heterologous expression systems. The differing approaches that have been used to analyze the effects of these mutations have lead to controversy, particularly in the case of the $\gamma 2^{R 43 Q}$ mutation (Petrou and Reid, 2012). Moreover, of all the identified epilepsycausing mutant $\mathrm{GABA}_{\mathrm{A}} \mathrm{Rs}$ that exhibit partial or full expression 
at the cell membrane, there is an animal knock-in model of only one (Petrou and Reid, 2012). Transgenic animal models are afflicted by compensatory mechanisms that can obfuscate data, especially those involving ion channel genetic manipulations that affect GABAergic transmission (Harris et al., 2011). Because coculture $\alpha 1 \beta 2 \gamma 2 \mathrm{GABA}_{\mathrm{A}} \mathrm{R}$ synapses successfully recapitulate the kinetics of neuronal IPSCs (Dixon et al., 2014), they may provide a promising means of investigating the synaptic signaling defects induced by hereditary epilepsy mutations to $\alpha 1$ and $\gamma 2$ subunits.

Glycinergic co-culture synapses incorporating $\alpha 1^{\mathrm{D} 80 \mathrm{~A} \beta}$ and $\alpha 1^{\text {A52S } \beta}$ GlyRs have been shown to exhibit accelerated IPSC decay rates that strongly resemble those recorded in native synapses from mutant mice homozygous for the same mutations (Zhang et al., 2014). This suggests that the co-culture system should be useful for modeling the effects of hyperekplexia mutations to $\alpha 1$ and $\beta$ subunits (Bode and Lynch, 2014) and autism mutations to $\alpha 2$ subunits (Pilorge et al., 2015). Here we investigated the effect of the $\alpha 3^{\mathrm{P} 185 \mathrm{~L}}$ mutation that is associated with temporal lobe epilepsy. The mutation resulted in slowing of the IPSC decay rate (Figure 3). However, glycinergic synapses are absent in the temporal lobe and it is thought that the affected receptors are located presynaptically at glutamatergic synapses. In this case, our results suggest the mutant GlyR would remain open for longer during each synaptic event, thus potentiating an excitatory $\mathrm{Cl}$ flux leading to enhanced excitatory neurotransmission that could underlie the disorder (Eichler et al., 2008, 2009; Legendre et al., 2009).

\section{Synaptogenesis and Synaptic Clustering Mechanisms}

Co-culture synapses have been used extensively to probe the roles of synaptic adhesion molecules in the formation of glutamatergic or GABAergic synapses (Scheiffele et al., 2000; Biederer et al., 2002; Dean et al., 2003; Graf et al., 2004; Sara et al., 2005; Kim et al., 2006; Dong et al., 2007; Fuchs et al., 2013) or to investigate the impact of disease-causing neuroligin mutations on GABAergic synaptogenesis (Chubykin et al., 2005; Sun et al., 2011). The strengths and weaknesses of co-cultures in this respect have recently been reviewed (Fuchs et al., 2013).

\section{CONCLUSION}

As the presynaptic terminals of our engineered synapses are provided by real neurons, their function is likely to resemble those of synapses in vivo. Indeed, serial electron microscopic reconstructions of GABAergic terminals onto HEK293 cells have confirmed that their ultrastructures are similar to those of native neurons (Fuchs et al., 2013). However, the postsynaptic specializations are less likely to resemble those of

\section{REFERENCES}

Biederer, T., Sara, Y., Mozhayeva, M., Atasoy, D., Liu, X., Kavalali, E. T., et al. (2002). SynCAM, a synaptic adhesion molecule that drives synapse assembly. Science 297, 1525-1531. doi: 10.1126/science.1072356 neurons given that HEK293 cells do not endogenously express all necessary postsynaptic clustering proteins at appropriate levels for synaptogenesis. In addition, some proteins that they do express may lack neuron-specific post-translational modifications required for correct synaptic function. These factors could ultimately alter the geometry of the synaptic cleft and the postsynaptic receptor clustering density, leading to non-physiological changes in the neurotransmitter concentration profile that could affect IPSC kinetics. This uncertainty is the main limitation of the technique. We have addressed this as far as possible by comparing the properties of engineered synapses with those of real synapses in cases where we can be reasonably sure about the synaptic subunit composition.

However, due to their non-physiological status, engineered synapses also offer opportunities to investigate new clustering mechanisms. If, for example, substitution of a particular pLGIC subunit results in a drastic, unexpected slowing of the IPSC rise time, it is possible that synaptic receptors have been de-clustered in a manner that may not occur in a neuron. This could in turn lead to the identification of novel clustering molecules and mechanisms. HEK293 cells are ideal for investigating such questions: they do not express all proteins necessary for synaptogenesis, but they do provide a high efficiency of transfection, faithful protein translation and a small, electronically compact shape appropriate for accurate quantitation of IPSC rise and decay times (Thomas and Smart, 2005).

\section{AUTHOR CONTRIBUTIONS}

$\mathrm{CD}, \mathrm{YZ}$, and JL conceived the project and developed the protocols; $\mathrm{CD}$ and $\mathrm{YZ}$ performed experiments and analyzed the data; and CD, YZ, and JL wrote the manuscript.

\section{ACKNOWLEDGMENTS}

This research was supported by project grants from the Australian Research Council (DP120104373) and the National Health and Medical Research Council (APP1062183). JL is supported by a Principle Research Fellowship from the National Health and Medical Research Council (APP1058542). We thank Dr Nela Durisic for critical review of the manuscript.

\section{SUPPLEMENTARY MATERIAL}

The Supplementary Material for this article can be found online at: http://journal.frontiersin.org/article/10.3389/fnmol. 2015.00080

Biederer, T., and Scheiffele, P. (2007). Mixed-culture assays for analyzing neuronal synapse formation. Nat. Protoc. 2, 670-676. doi: 10.1038/nprot.2007.92

Bode, A., and Lynch, J. W. (2014). The impact of human hyperekplexia mutations on glycine receptor structure and function. Mol. Brain 7, 2. doi: 10.1186/17566606-7-2 
Brewer, G. J. (1995). Serum-free B27/neurobasal medium supports differentiated growth of neurons from the striatum, substantia nigra, septum, cerebral cortex, cerebellum, and dentate gyrus. J. Neurosci. Res. 42, 674-683. doi: 10.1002/jnr.490420510

Brewer, G. J., Torricelli, J. R., Evege, E. K., and Price, P. J. (1993). Optimized survival of hippocampal neurons in B27-supplemented Neurobasal, a new serum-free medium combination. J. Neurosci. Res. 35, 567-576. doi: 10.1002/jnr.490350513

Brown, L. E., Fuchs, C., Nicholson, M. W., Stephenson, F. A., Thomson, A. M., and Jovanovic, J. N. (2014). Inhibitory synapse formation in a co-culture model incorporating GABAergic medium spiny neurons and HEK293 cells stably expressing GABAA receptors. J. Vis. Exp. 93, e52115. doi: 10.3791/52115

Chubykin, A. A., Liu, X., Comoletti, D., Tsigelny, I., Taylor, P., and Sudhof, T. C. (2005). Dissection of synapse induction by neuroligins: effect of a neuroligin mutation associated with autism. J. Biol. Chem. 280, 22365-22374. doi: $10.1074 /$ jbc.M410723200

Dean, C., Scholl, F. G., Choih, J., DeMaria, S., Berger, J., Isacoff, E., et al. (2003). Neurexin mediates the assembly of presynaptic terminals. Nat. Neurosci. 6, 708-716. doi: 10.1038/nn1074

Dixon, C., Sah, P., Lynch, J. W., and Keramidas, A. (2014). GABAA receptor alpha and gamma subunits shape synaptic currents via different mechanisms. J. Biol. Chem. 289, 5399-5411. doi: 10.1074/jbc.M113.514695

Dixon, C. L., Harrison, N. L., Lynch, J. W., and Keramidas, A. (2015). Zolpidem and eszopiclone prime alphalbeta2gamma2 GABAA receptors for longer duration of activity. Br. J. Pharmacol. 172, 3522-3536. doi: 10.1111/bph.13142

Dong, N., Qi, J., and Chen, G. (2007). Molecular reconstitution of functional GABAergic synapses with expression of neuroligin-2 and GABAA receptors. Mol. Cell. Neurosci. 35, 14-23. doi: 10.1016/j.mcn.2007.01.013

Durisic, N., Godin, A. G., Wever, C. M., Heyes, C. D., Lakadamyali, M., and Dent, J. A. (2012). Stoichiometry of the human glycine receptor revealed by direct subunit counting. J. Neurosci. 32, 12915-12920. doi: 10.1523/JNEUROSCI.2050-12.2012

Eichler, S. A., Forstera, B., Smolinsky, B., Juttner, R., Lehmann, T. N., Fahling, M., et al. (2009). Splice-specific roles of glycine receptor alpha3 in the hippocampus. Eur. J. Neurosci. 30, 1077-1091. doi: 10.1111/j.1460-9568.2009.06903.x

Eichler, S. A., Kirischuk, S., Juttner, R., Schaefermeier, P. K., Legendre, P., Lehmann, T. N., et al. (2008). Glycinergic tonic inhibition of hippocampal neurons with depolarizing GABAergic transmission elicits histopathological signs of temporal lobe epilepsy. J. Cell. Mol. Med. 12, 2848-2866. doi: 10.1111/j.15824934.2008.00357.x

Fuchs, C., Abitbol, K., Burden, J. J., Mercer, A., Brown, L., Iball, J., et al. (2013). GABA(A) receptors can initiate the formation of functional inhibitory GABAergic synapses. Eur. J. Neurosci. 38, 3146-3158. doi: 10.1111/ejn.12331

Graf, E. R., Zhang, X., Jin, S. X., Linhoff, M. W., and Craig, A. M. (2004). Neurexins induce differentiation of GABA and glutamate postsynaptic specializations via neuroligins. Cell 119, 1013-1026. doi: 10.1016/j.cell.2004.11.035

Graham, B. A., Schofield, P. R., Sah, P., Margrie, T. W., and Callister, R. J. (2006). Distinct physiological mechanisms underlie altered glycinergic synaptic transmission in the murine mutants spastic, spasmodic, and oscillator. J. Neurosci. 26, 4880-4890. doi: 10.1523/JNEUROSCI.3991-05.2006

Harris, R. A., Osterndorff-Kahanek, E., Ponomarev, I., Homanics, G. E., and Blednov, Y. A. (2011). Testing the silence of mutations: transcriptomic and behavioral studies of GABA(A) receptor alpha1 and alpha2 subunit knock-in mice. Neurosci. Lett. 488, 31-35. doi: 10.1016/j.neulet.2010.10.075

Harvey, R. J., Depner, U. B., Wassle, H., Ahmadi, S., Heindl, C., Reinold, H., et al. (2004). GlyR alpha3: an essential target for spinal PGE2-mediated inflammatory pain sensitization. Science 304, 884-887. doi: 10.1126/science.1094925

Hirzel, K., Muller, U., Latal, A. T., Hulsmann, S., Grudzinska, J., Seeliger, M. W., et al. (2006). Hyperekplexia phenotype of glycine receptor alphal subunit mutant mice identifies $\mathrm{Zn}(2+)$ as an essential endogenous modulator of glycinergic neurotransmission. Neuron 52, 679-690. doi: 10.1016/j.neuron.2006.09.035

Kim, S., Burette, A., Chung, H. S., Kwon, S. K., Woo, J., Lee, H. W., et al. (2006). NGL family PSD-95-interacting adhesion molecules regulate excitatory synapse formation. Nat. Neurosci. 9, 1294-1301. doi: 10.1038/nn1763

Knoflach, F., Benke, D., Wang, Y., Scheurer, L., Luddens, H., Hamilton, B. J., et al. (1996). Pharmacological modulation of the diazepam-insensitive recombinant gamma-aminobutyric acidA receptors alpha 4 beta 2 gamma 2 and alpha 6 beta 2 gamma 2. Mol. Pharmacol. 50, 1253-1261.
Legendre, P., Forstera, B., Juttner, R., and Meier, J. C. (2009). Glycine Receptors Caught between genome and proteome - functional implications of RNA editing and splicing. Front. Mol. Neurosci. 2:23. doi: 10.3389/neuro.02.023.2009

Lynagh, T., and Lynch, J. W. (2010). A glycine residue essential for high ivermectin sensitivity in Cys-loop ion channel receptors. Int. J. Parasitol. 40, 1477-1481. doi: 10.1016/j.jpara.2010.07.010

Lynch, J. W. (2009). Native glycine receptor subtypes and their physiological roles. Neuropharmacology 56, 303-309. doi: 10.1016/j.neuropharm.2008.07.034

Lynch, J. W., and Callister, R. J. (2006). Glycine receptors: a new therapeutic target in pain pathways. Curr. Opin. Investig. Drugs 7, 48-53.

Macdonald, R. L., Kang, J. Q., and Gallagher, M. J. (2010). Mutations in GABAA receptor subunits associated with genetic epilepsies. J. Physiol. 588, 1861-1869. doi: 10.1113/jphysiol.2010.186999

Meier, J. C., Henneberger, C., Melnick, I., Racca, C., Harvey, R. J., Heinemann, U., et al. (2005). RNA editing produces glycine receptor alpha3(P185L), resulting in high agonist potency. Nat. Neurosci. 8, 736-744. doi: 10.1038/nn1467

Muller, E., Le Corronc, H., Triller, A., and Legendre, P. (2006). Developmental dissociation of presynaptic inhibitory neurotransmitter and postsynaptic receptor clustering in the hypoglossal nucleus. Mol. Cell. Neurosci. 32, 254-273. doi: 10.1016/j.mcn.2006.04.007

Olsen, R. W., and Sieghart, W. (2009). GABA A receptors: subtypes provide diversity of function and pharmacology. Neuropharmacology 56, 141-148. doi: 10.1016/j.neuropharm.2008.07.045

Petrou, S., and Reid, C. A. (2012). The GABAAgamma2(R43Q) Mouse Model of Human Genetic Epilepsy. Parkville VIC: Florey Neuroscience Institute and The Centre for Neuroscience, The University of Melbourne.

Pilorge, M., Fassier, C., Le Corronc, H., Potey, A., Bai, J., De Gois, S., et al. (2015). Genetic and functional analyses demonstrate a role for abnormal glycinergic signaling in autism. Mol. Psychiatry doi: 10.1038/mp.2015.139 [Epub ahead of print].

Qi, Z.-H., Song, M., Wallace, M. J., Wang, D., Newton, P. M., McMahon, T., et al. (2007). Protein kinase $C \epsilon$ regulates $\gamma$-aminobutyrate type A receptor sensitivity to ethanol and benzodiazepines through phosphorylation of $\gamma 2$ subunits. J. Biol. Chem. 282, 33052-33063. doi: 10.1074/jbc.M707233200

Sara, Y., Biederer, T., Atasoy, D., Chubykin, A., Mozhayeva, M. G., Sudhof, T. C., et al. (2005). Selective capability of SynCAM and neuroligin for functional synapse assembly. J. Neurosci. 25, 260-270. doi: 10.1523/JNEUROSCI.316504.2005

Scheiffele, P., Fan, J., Choih, J., Fetter, R., and Serafini, T. (2000). Neuroligin expressed in nonneuronal cells triggers presynaptic development in contacting axons. Cell 101, 657-669. doi: 10.1016/S0092-8674(00)80877-6

Singer, J. H., Talley, E. M., Bayliss, D. A., and Berger, A. J. (1998). Development of glycinergic synaptic transmission to rat brain stem motoneurons. J. Neurophysiol. 80, 2608-2620.

Soh, M. S., and Lynch, J. W. (2015). Selective modulators of alpha5-containing GABAA receptors and their therapeutic significance. Curr. Drug Targets 16, 735-746. doi: 10.2174/1389450116666150309120235

Sun, C., Cheng, M. C., Qin, R., Liao, D. L., Chen, T. T., Koong, F. J., et al. (2011). Identification and functional characterization of rare mutations of the neuroligin-2 gene (NLGN2) associated with schizophrenia. Hum. Mol. Genet. 20, 3042-3051. doi: 10.1093/hmg/ddr208

Talwar, S., and Lynch, J. W. (2014). Phosphorylation mediated structural and functional changes in pentameric ligand-gated ion channels: implications for drug discovery. Int. J. Biochem. Cell Biol. 53, 218-223. doi: 10.1016/j.biocel.2014.05.028

Thomas, P., and Smart, T. G. (2005). HEK293 cell line: a vehicle for the expression of recombinant proteins. J. Pharmacol. Toxicol. Methods 51, 187-200. doi: 10.1016/j.vascn.2004.08.014

Trincavelli, M. L., Da Pozzo, E., Daniele, S., and Martini, C. (2012). The GABAABZR complex as target for the development of anxiolytic drugs. Curr. Top. Med. Chem. 12, 254-269. doi: 10.2174/1568026799078787

Wallner, M., Hanchar, H. J., and Olsen, R. W. (2006). Low dose acute alcohol effects on GABA A receptor subtypes. Pharmacol. Ther. 112, 513-528. doi: 10.1016/j.pharmthera.2006.05.004

Wu, X., Wu, Z., Ning, G., Guo, Y., Ali, R., Macdonald, R. L., et al. (2012). gammaAminobutyric acid type A (GABAA) receptor alpha subunits play a direct role in synaptic versus extrasynaptic targeting. J. Biol. Chem. 287, 27417-27430. doi: $10.1074 /$ jbc.M112.360461 
Yang, Z., Taran, E., Webb, T. I., and Lynch, J. W. (2012). Stoichiometry and subunit arrangement of alphalbeta glycine receptors as determined by atomic force microscopy. Biochemistry 51, 5229-5231. doi: 10.1021/bi $300063 \mathrm{~m}$

Zeilhofer, H. U. (2005). The glycinergic control of spinal pain processing. Cell. Mol. Life Sci. 62, 2027-2035. doi: 10.1007/s00018-005$5107-2$

Zhang, Y., Dixon, C. L., Keramidas, A., and Lynch, J. W. (2014). Functional reconstitution of glycinergic synapses incorporating defined glycine receptor subunit combinations. Neuropharmacology 89C, 391-397.
Conflict of Interest Statement: The authors declare that the research was conducted in the absence of any commercial or financial relationships that could be construed as a potential conflict of interest.

Copyright $\odot 2015$ Dixon, Zhang and Lynch. This is an open-access article distributed under the terms of the Creative Commons Attribution License (CC BY). The use, distribution or reproduction in other forums is permitted, provided the original author(s) or licensor are credited and that the original publication in this journal is cited, in accordance with accepted academic practice. No use, distribution or reproduction is permitted which does not comply with these terms. 